\title{
Simultaneous Operations in Gynecology and Surgery
}

\author{
Dronova V, Dronov O
}

\begin{abstract}
Public institute of "Institute of pediatrics, obstetrics and gynecology AMS of Ukraine"
\section{INTRODUCTION}

Numerous studies indicate widespread combined surgical diseases of the abdomen and pelvis, are diagnosed more than $60 \%$ patients of surgical hospitals. However, only in $11-17 \%$ of cases performed simultaneous intervention. Surgical treatment of combined surgical gynecologic and extragenital diseases involves the use of combined operational approaches that can eliminate the detected pathology under one anesthesia, eliminating the need to repeat procedures and related complications, improves quality of life (physical activity, mental state, social and sexual functioning).
\end{abstract}

\section{THE OBJECT OF THE STUDY}

To improve the results of surgical treatment of women with gynecological and extragenital combined surgical pathology using standardized diagnostic algorithm based on diagnostic and tactical definition neurohormonal parameters "surgical stress."

\section{OBJECT AND METHODS}

During the years 2013-2014 in the department of operative gynecology and surgical clinics of general surgery operated on 335 women, of which $155(46,3 \%)$ were carrying out simultaneous interventions on the concomitant extragenital surgical pathology. The department of operative gynecology completed $290(71,4 \%)$ operations $102(65,8 \%)$ women in the clinics of general surgery - 116 $(28,6 \%)$ operations $53(34,2 \%)$ patients.

To determine the neuroendocrine system changes in patients' response to surgical trauma stress indicators studied hormonal activity in pre- and postoperative period, prolactin, cortisol, thyroidstimulating hormone (TSH), thyroxine T4 and melatonin.

Simultaneous surgeries were performed in patients aged 14 to 75 years, and $12,5 \%$ - women over 60 years with concomitant somatic pathology. In total 155 women was conducted 406 interventions, with one woman held from 2 to 6 surgeries in conditions of one anesthesia. The amount of interference in each woman individually determined according to the detected pathology. Among the most frequently performed surgery combined, hysterectomy with cholecystectomy - $20(13,5 \%)$ and transactions with the elimination of partial intestinal obstruction - $12(8,1 \%)$, removal of ovarian cysts cholecystectomy - $21(14,2 \%)$, hysterectomy with vein removal - $10(10,8 \%)$.

\section{RESULTS AND DISCUSSION}

Determination of stress-related hormones in the blood serum of women surveyed performed before surgery, the 1st and 3rd day after surgery. Groups of women were compared, which only performed gynecological surgery (1st group) and women who carried out simultaneous intervention (2nd group). The study did not reveal significant changes in the average concentrations of prolactin and cortisol, they do not go beyond reference standards in both groups. The study of thyroid parameters TSH and T4 found that the significant difference in women first and second group was not. The concentration of melatonin in the blood serum of the first group of women to 2-2.5 times the performance of the second group of women pre- and in the first days after surgery. In the second group of women this figure at all stages of examination was within normal limits with a certain tendency to increase on the third day after surgery.

\section{CONCLUSION}

In preparing women with diseases of pelvic surgery advisable to extend the standard preoperative examination for the purpose of diagnosis combined extra-surgical diseases and execution of simultaneous operations. Determination of stress-related hormones (cortisol, prolactin) in the serum of women surveyed are not reliable and indicative setting displays surgical stress. Melatonin as a universal adaptogen is exemplary in carrying out simultaneous operations on the third day postoperative period and may be a diagnostic marker of recovery and normalization of the patient. 